\title{
Cerebral small vessel disease and Alzheimer's disease
}

This article was published in the following Dove Press journal:

Clinical Interventions in Aging

23 October 2015

Number of times this article has been viewed

\section{Zhiyou Cai' \\ Chuanling Wang' \\ Wenbo $\mathrm{He}^{1}$ \\ Hanjun $\mathrm{Tu}^{2}$ \\ Zhengang Tang ${ }^{3}$ \\ Ming Xiao ${ }^{4}$ \\ Liang-Jun Yan ${ }^{5}$}

'Department of Neurology, Renmin Hospital, Hubei University of Medicine, ${ }^{2}$ Department of Basic Research Center, Hubei University of Medicine, ${ }^{3}$ Department of Neurosurgery, Renmin Hospital, Hubei University of Medicine, Shiyan, Hubei Province, ${ }^{4}$ Department of Anatomy, Nanjing Medical University, Nanjing, Jiangsu, People's Republic of China; ${ }^{5}$ Department of Pharmaceutical Sciences, UNT System College of Pharmacy, University of North Texas Health Science Center, Fort Worth, TX, USA
Correspondence: Zhiyou Cai Department of Neurology, Renmin Hospital, Shiyan Renmin Hospital, Hubei University of Medicine, No 39 Chaoyang Middle Road, Shiyan, Hubei Province 442000, People's Republic of China Tel/fax +86 7198637909

Email c0909@hotmail.com
Abstract: Cerebral small vessel disease (CSVD) is a group of pathological processes with multifarious etiology and pathogenesis that are involved into the small arteries, arterioles, venules, and capillaries of the brain. CSVD mainly contains lacunar infarct or lacunar stroke, leukoaraiosis, Binswanger's disease, and cerebral microbleeds. CSVD is an important cerebral microvascular pathogenesis as it is the cause of $20 \%$ of strokes worldwide and the most common cause of cognitive impairment and dementia, including vascular dementia and Alzheimer's disease (AD). It has been well identified that CSVD contributes to the occurrence of AD. It seems that the treatment and prevention for cerebrovascular diseases with statins have such a role in the same function for AD. So far, there is no strong evidence-based medicine to support the idea, although increasing basic studies supported the fact that the treatment and prevention for cerebrovascular diseases will benefit AD. Furthermore, there is still lack of evidence in clinical application involved in specific drugs to benefit both AD and CSVD.

Keywords: dementia, cerebrovascular diseases, lacunar infarct, leukoaraiosis, cerebral microbleeds

\section{Introduction}

Alzheimer's disease (AD) is a progressive neurodegenerative disease of the brain. AD is clinically characterized by cognitive impairment at first and dementia eventually, which gradually worsen over a number of years. ${ }^{1}$ Increasing research indicates that AD results from the destruction and death of nerve cells triggered by amyloid plaques and neurofibrillary tangles, ${ }^{1-3}$ both of which are thought to contribute to the neurodegenerative death of the neurons in the brain and the subsequent symptoms of AD. The main risk factor for $\mathrm{AD}$ is advanced age, while the incidence of $\mathrm{AD}$ increases with the progress of aging. ${ }^{4}$ Substantial clinical research implicates the involvement of vascular risk factors in $\mathrm{AD}$, such as hypertension, ${ }^{5}$ coronary artery disease,${ }^{6}$ diabetes, ${ }^{7,8}$ and hyperlipidemia. ${ }^{9,10}$ These vascular factors increase the risk of $\mathrm{AD}$ occurrence..$^{5,11}$

Cerebral small vessel disease (CSVD) is considered as an important pathological process of subcortical structures such as lacunar infarcts, white matter lesions, and microbleeds. ${ }^{12}$ CSVD is responsible for the pathogenesis of ischemic strokes, cerebral hemorrhages, and encephalopathy, which are associated with advanced age, and deteriorated by hypertension and diabetes mellitus. ${ }^{13}$ CSVD mainly contains lacunar infarct or lacunar stroke, leukoaraiosis, Binswanger's disease, and cerebral microbleeds. ${ }^{11} \mathrm{With}$ the development of imaging examination and its clinical usage, it has been well identified that cerebral vascular diseases, especially CSVD, contribute to the occurrence of $\mathrm{AD}$ (Figure 1). ${ }^{9,14}$

This work focused on the relationship between AD and CSVD. An overview was provided for the association between $\mathrm{AD}$ and lacunar infarct, leukoaraiosis, 


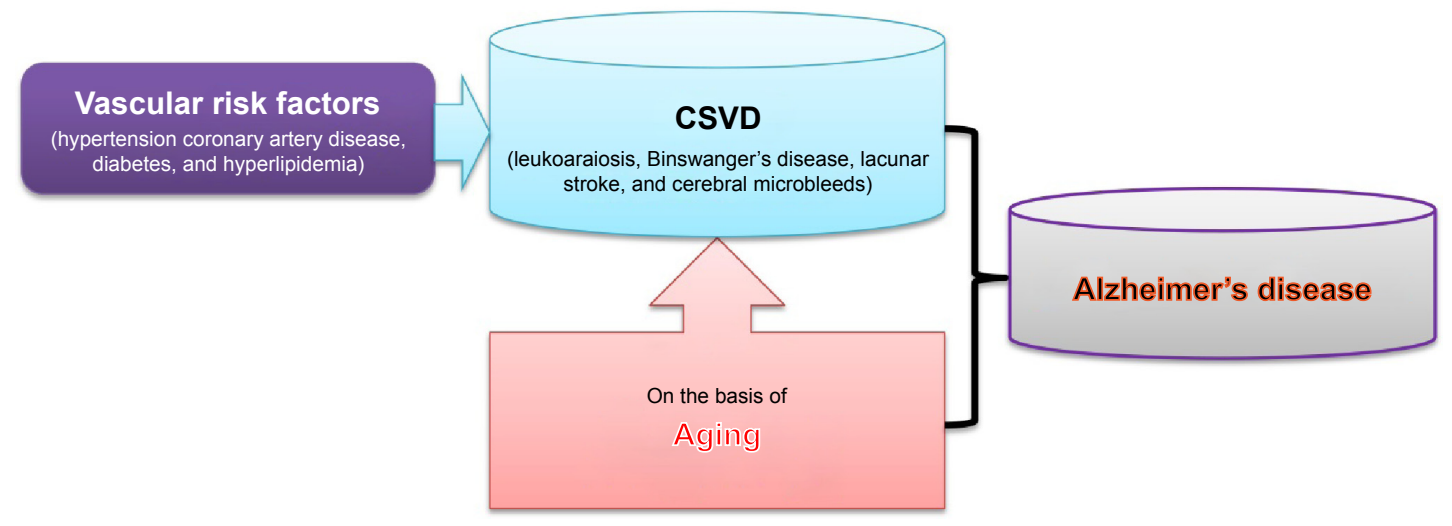

Figure I CSVD and Alzheimer's disease.

Notes: Both CSVD and aging contribute to the occurrence of Alzheimer's disease. On the basis of aging, CSVD can accelerate the development of Alzheimer's disease. Abbreviation: CSVD, cerebral small vessel disease.

Binswanger's disease, and cerebral microbleeds, respectively. It was reviewed that CSVD plays an important role in the development of cognitive impairment and dementia. It was also discussed that it is possibly an effective measure to prevent cerebrovascular disorders as an attractive target for AD.

\section{Cerebral small vessel disease}

CSVD, microangiopathy of the cerebral white and gray matter, indicates a group of pathological process with multifarious etiology and pathogenesis that involve the small vessels, including arterioles, venules, and capillaries of the brain. ${ }^{15,16}$ CSVD is responsible for $20 \%-30 \%$ cases of ischemic strokes, as well as for a considerable proportion of cerebral hemorrhages and encephalopathy. ${ }^{17} \mathrm{CSVD}$ is regarded as an important pathological process of subcortical structures such as lacunar infarcts, white matter lesions, and microbleeds. ${ }^{12}$ Despite atherosclerotic plaque biology has not been considered into being suitable to small cerebral vessels, a lesion by fibrinoid necrosis causing lacunar infarcts and primary intracerebral bleeds has been identified as an important pathology in the CSVD. ${ }^{18}$ CSVD is a common aging phenomenon that is exacerbated by hypertension and diabetes mellitus, ${ }^{13}$ including leukoaraiosis, Binswanger's disease, lacunar stroke, and cerebral microbleeds. ${ }^{19}$ Leukoaraiosis refers to white matter changes (WMC) in the brain, often occurring after 65 years of age. Leukoaraiosis pathogenesis includes breakdown of axons, an unnatural paleness of myelin, gliosis, loss of ependymal cells, and enlarged perivascular spaces. Binswanger's disease is also known as subcortical leukoencephalopathy caused by arteriosclerosis and thromboembolism influencing the blood vessels on the blood supply of white matter. Lacunar stroke or lacunar infarct is a little stroke that is caused from occlusion of the penetrating small arteries that supply blood into the brain's deep distributions. Cerebral microbleeds result from impaired small vessel integrity, mainly being attributed to either hypertensive vasculopathy or cerebral amyloid angiopathy. Microbleeds are commonly combined with Alzheimer's dementia and stroke. ${ }^{20}$ Research findings demonstrate that vascular factors play a pathogenic role in the early stages of $\mathrm{AD}$ because these cerebrovascular alterations impair the delicate balance between the brain's energy requirements and cerebral blood supply, ${ }^{21-23}$ resulting in the upregulation of beta-amyloid $(\mathrm{A} \beta)$ production and impaired $A \beta$ drainage. ${ }^{24,25}$

Age-related and hypertension-related small vessel diseases are the most common forms of CSVD (Figure 1). ${ }^{15}$ CSVD is often recognized as the most common etiology of vascular cognitive impairment and dementia, acting as the same role of large vessel disease and other forms of cerebrovascular disease (CVD) in vascular cognitive impairment and dementia. CSVD gradually develops into decline of cognition, vascular dementia (VaD), impairment of gait and balance, mood depression, and urinary incontinence and often results in great social and economic burdens. ${ }^{17}$ Increasing evidences suggest that there are decreased vascular density and cerebral microvascular pathology in the process of aging and neurodegeneration and cerebrovascular dysfunction, and CSVD precedes or accompanies cognitive dysfunction and neurodegeneration. ${ }^{26}$ Several investigations indicate that cerebrovascular atherosclerosis, especially atherosclerosis of the Willis circle, is more severe in AD and correlates with the severity of AD pathology, ${ }^{27,28}$ implicating that atherosclerosisinduced cerebrovascular hypoperfusion correlates with $\mathrm{AD}$ pathology and the clinical manifestations of AD. CSVD on the basis of atherosclerosis will accelerate the development of 
AD since cerebral hypoperfusion and reduced glucose uptake have been found very early in $\mathrm{AD},{ }^{28,29}$ in consideration that the regulation of cerebral blood flow plays an important role in the coordinated interaction of neurons, glia, and vascular cells. Many studies show that CSVD increases the risk of AD occurrence, while signs of AD pathology can often coexist with both $\mathrm{VaD}$ and CSVD. ${ }^{30}$

\section{Lacunar infarct and AD}

Lacunar infarct or lacunar stroke is a small $(0.2-15 \mathrm{~mm}$ in diameter) noncortical infarct, which is generally believed to be the cause of occlusion of the penetrating arteries. Lacunar infarct onsets when one of the penetrating arteries that provide blood to the brain's deep distributions is obstructed. It is assessed that lacunar infarcts make up $25 \%$ of all ischemic strokes, with an annual incidence of approximately 15 per 100,000 people. ${ }^{31,32}$ The clinical manifestations of lacunar stroke vary, such as the disorders of sensation, movement, sight, speech, balance, and coordination, because different functions are determined by different areas of the brain involved in lacunar stroke. ${ }^{8}$ Diagnosis of lacunar stroke is mainly according to the setting of clinical representations and neuroimaging results. ${ }^{33}$ In many cases, a lacunar infarct manifests a silent stroke, which does not have any outside symptoms. Patients are not aware of it at all when they suffer a lacunar stroke. Lacunar infarct induces a significant impairment to the cells in small parts of the brain and leads to death of corresponding parts of the brain tissue. In spite of no symptoms, lacunar stroke still gradually destroys the brain function and acts as an existing risk of major stroke attacks therein. $^{34,35}$ The onset of multiple lacunar strokes occur and get accumulated with the progress of aging and role of risk factors. This ultimately results in a state of cognitive impairment and development of dementia. ${ }^{36-38}$

It is well accepted that lacunar infarct is an age-related cerebral ischemia disorder, ${ }^{39,40}$ and the different location of lacunar infarct within subcortical gray matter is a determinant of different cognitive impairment. ${ }^{40,41}$ The greatest known risk factor of $\mathrm{AD}$ is the advancing age, and the majority of patients with $\mathrm{AD}$ are $>60$ years old. ${ }^{42}$ Accordingly, aging is a common contributor to the occurrence of lacunar infarct and the development of AD. Furthermore, AD and lacunar infarct have a considerable degree of overlap in risk factors, ${ }^{43-45}$ such as hypertension, diabetes, hyperlipidemia, adiposity, smoking, and drinking, as well as unhealthy lifestyle.

A number of clinical and basic findings indicate that CVD is closely associated with both the presence and the severity of the clinical symptoms of AD. ${ }^{46}$ As the progress of cerebrovascular lesions was inextricably linked with vascular risk factors and age, the involvement of vascular risk factors will fully aid in clinical presentation of elderly patients with $\mathrm{AD}{ }^{42,47}$ It has been found that $\mathrm{AD}$ is highly prevalent in the elderly and often coexists with multimicroinfarcts; the number of microinfarcts is roughly in proportion to the degree of cognitive impairment, and the presence of microinfarcts was intimately linked with AD rather than cerebral amyloid angiopathy. ${ }^{48}$ Lacunar infarct, one of the cerebrovascular disorders, is also known to play important roles in the pathogenesis underlying $\mathrm{AD}$, especially in the elderly patients with AD. ${ }^{42}$ Additionally, several studies implicate the associations between lacunar infarct and Alzheimer's pathology, including $A \beta$ and tau pathology. ${ }^{49-51} \mathrm{~A}$ crosssectional study showed that the presence of lacunar infarct was associated with higher $\mathrm{A} \beta 42$ in $\mathrm{VaD}$ and lower tau in $\mathrm{AD}$ in cerebrospinal fluid (CSF). ${ }^{49}$ Another cross-sectional study demonstrated that the presence of lacunar infarctions could be tightly coupled to increased plasma $A \beta 40$ and plasma A $\beta 40$ concentration in the patients with AD. ${ }^{51}$

However, the relationship between $\mathrm{AD}$ and lacunar infarct remains largely unclear. Although it appears that there are several shares in risk factors and related pathogenesis in AD and lacunar infarct, how these neglected silent infarcts develop multiple domain cognitive deficits and AD? It seems that cerebral atherosclerosis plays a strong role in the occurrence of cystic infarcts and microinfarcts but not Alzheimer's pathogenesis. ${ }^{9}$

\section{Cerebral microbleeds and AD}

Currently, cerebral microbleeds have been well identified as an important imaging marker of CSVD. Cerebral microbleeds are small hypointense imaging appearances according to $\mathrm{T} 2 *$ gradient-recall echo and susceptibility-weighted magnetic resonance imaging (MRI) sequences. ${ }^{20,52,53} \mathrm{With}$ the development of MRI techniques, cerebral microbleeds have been found in the patients of stroke and cognitive impairment, as well as in healthy people and in people with other disorders. ${ }^{54,55}$ A confirmed diagnosis of cerebral microbleeds indicates the acceptance of the underlying small vessel disease, the safety of antithrombotic use, the risk of symptomatic intracerebral hemorrhage, and the cause of cognitive impairment and dementia. ${ }^{56,57}$ Substantial literature to date has indicated that cerebral microbleeds contribute to the clinical manifestation and are linked with the occurrence of biochemical hallmarks of $\mathrm{AD}$, suggesting the involvement of cerebral microbleeds in the pathogenesis of $\mathrm{AD} .^{58}$ 
Multiple cerebral microbleeds have been shown to occur in $\mathrm{AD}$ and are linked with cerebral network breakdowns in the patients with $\mathrm{AD},{ }^{59}$ affecting approximately one-third of subjects who are present with the clinical manifestations of AD dementia. ${ }^{60}$ Furthermore, cerebral microbleeds in AD are more closely linked with cerebral amyloid angiopathy than CVD. ${ }^{61}$ Atypical AD subjects seem to be at a particular risk for developing large numbers of microbleeds and for developing microbleeds in the frontal lobe under T2*-weighted MRI. ${ }^{60,62}$ In a review literature, it has been proved that the neuropathology of AD results in the breakdown of the silent microbleeds, caused by pulse-induced damage to the cerebral vessels underlying the age-related stiffening of the aorta and great arteries, which increases the intensity of the pressure pulse. ${ }^{63}$ It has been reported that the incidence of multiple lobar microbleeds that have occurred in the preclinical $\mathrm{AD}$ and microbleeds in $\mathrm{AD}$ are particularly linked with additional $\mathrm{A} \beta$ burden. ${ }^{64}$ Cerebral microbleeds were also related to the alterations of $\mathrm{A} \beta$ metabolism in $\mathrm{AD} .{ }^{65}$ Concomitantly, older age, higher $\mathrm{A} \beta$ burden, and CVD may all facilitate the occurrence of lobar microbleeds. ${ }^{66}$ A crosssectional study, investigating the prevalence, locations, and risk factors for cerebral microbleeds in neurodegenerative disorders identified that cerebral microbleeds are linked with vascular burden and AD diagnosis. ${ }^{67}$ It has been reported that there was a high prevalence of cerebral microbleeds in patients with early AD according to 7 T MRI tests. ${ }^{68}$

A growing body of research studies has proved that sortilin-related receptor 1 gene (SORL1) is genetically linked to AD pathological changes. ${ }^{69-73}$ The SORL1 was downregulated in the AD brain and positively contributed to $\mathrm{A} \beta$ accumulation. Emerging data suggest that SORL1 acts as a central regulating role of the trafficking and processing of amyloid precursor protein and interacts with ApoE and tau protein. ${ }^{71}$ A recent study found that SORL1 single nucleotide polymorphism (SNP) rs2070045-G allele was linked to CSF-tau and hippocampal atrophy, two endophenotype markers of AD. ${ }^{74}$ In addition, haplotype-based analyses discovered an association between haplotype rs11218340-A/rs3824966-G/rs3824968-A and higher CSF-tau and CSF-tau phosphorylated at threonine 181, implicating that SORL1 may be the tau pathology in AD. ${ }^{74}$ The association of SORL1 with cerebral microbleeds indicates that the amyloid cascade sweeps into microbleeds into the etiology of AD. ${ }^{75}$

Cerebral microbleeds have been highlighted as a potential key risk factor in the pathogenesis of $\mathrm{AD}$, linking the main pathological contributors of $A \beta$ accumulation with cerebrovascular damage. ${ }^{76}$ However, it seems that cerebral microbleeds are likely to unfavorably affect cognitive functioning. ${ }^{77}$ There is a debate concerning the relationship between microangiopathy and the clinical course of AD or the conversion of mild cognitive impairment to AD. ${ }^{78,79}$ Cerebral microbleeds did not affect disease course in terms of progression to AD. ${ }^{77,80}$ Hence, the role of cerebral microbleeds in AD remains to be identified.

\section{Leukoaraiosis and AD}

Leukoaraiosis describes a diffuse, aberrant appearance of white matter on imaging, often presented in the normal elderly and in the patients with vascular risk factors or those suffering with cognitive impairment. ${ }^{81,82}$ The most common imaging to find leukoaraiosis is hyperintensity on T2-weighted MR in cerebral white matter. Age, hypertension, diabetes mellitus, and cardiovascular diseases are the major risk factors for leukoaraiosis. ${ }^{83}$ Leukoaraiosis was characterized by reduced cerebral blood flow and cerebrovascular reactivity and a leakage in the blood-brain barrier. ${ }^{84}$ It is gradually becoming clearer that leukoaraiosis is associated with $\mathrm{CVD},{ }^{85} \mathrm{AD}$, and other diseases. ${ }^{83}$

It has been reported that in compelling neuroimaging data, similar white matter abnormalities of leukoaraiosis appear in the patients with $\mathrm{AD}$ and in elderly healthy people. ${ }^{85-88}$ Coffman et al confirmed that the presence of leukoaraiosis might be of important significance in understanding changes in the white matter among populations at increased risk for AD ${ }^{89}$ A longitudinal study indicated that leukoaraiosis is associated with a greater degree of cognitive impairment in patients with $\mathrm{AD} .{ }^{90} \mathrm{~A}$ cross-sectional, descriptive, multicenter study involving 109 patients with AD and 59 with mild cognitive impairment identified that $\mathrm{AD}$ had a higher prevalence of leukoaraiosis and apathy. ${ }^{91}$ A recent pilot study suggests that jugular venous reflux is associated with cerebral WMC in individuals with AD, implying that cerebral venous outflow impairment might play a role in the dynamics of WMC in patients with AD, particularly in the periventricular regions. ${ }^{92}$ It is well accepted that white matter hyperintensities $(\mathrm{WMH})$ in imaging are linked with aging and $\mathrm{AD} .^{93}$ Makedonov et al reported that WMH perfusion was lower than normal-appearing white matter perfusion in both agematched elderly controls and AD groups. WMH tended to have a lower perfusion in AD compared with age-matched elderly. ${ }^{94}$ This imaging study suggests a common WMH etiology in AD and healthy aging. This finding is also suggestive of susceptible WMH hypoperfusion in AD compared with healthy aging, implicating that AD pathology decreases the perfusion at anatomic locations susceptible to the formation of $\mathrm{WMH}$ through either the neurodegenerative process or AD-related vasculopathy or both. ${ }^{94}$ The leukoaraiosis and disability prospective multinational European study evaluated 
the influence of WMC on the transition of independent elderly subjects, disclosing that self-perceived memory complaints predicted AD. ${ }^{95}$

\section{Binswanger's disease and AD}

In 1894, Dr Otto Binswanger, professor of psychiatry in Switzerland, first described a new clinical and neuropathological phenomenon that he termed "encephalitis subcorticalis chronica progressive" and Binswanger's disease is named after him. ${ }^{96,97}$ Other names for Binswanger's disease are also known as subcortical leukoencephalopathy, subcortical arteriosclerotic encephalopathy, ischemic periventricular leukoencephalopathy, or subcortical dementia. ${ }^{98-101}$ Binswanger's disease is featured by damage to small penetrating blood vessels in the subcortical regions of the brain. Binswanger's disease is often associated with advanced age, chronic hypertension, stroke, disease of the large blood vessels in the neck, alcohol and smoking, and cardiovascular diseases. Signs and symptoms of Binswanger's disease usually start after the age of 60 years. The onset of this disease is often between 54 years and 66 years of age, and the first symptoms are usually mental disorders or little stroke. A slow progressive dementia is identified as an important clinical feature of the disease. Binswanger's disease sometimes presents a rare form of dementia and often referred to as subcortical dementia.

Like other CSVD, Binswanger's disease shares some backgrounds with $\mathrm{AD},{ }^{102,103}$ including aging and vascular risk factors, such as hypertension, diabetes, cardiovascular diseases, metabolic syndrome, obesity, hyperlipidemia, smoking, and drinking. Binswanger's disease seems to be a risk factor for AD. ${ }^{104}$ However, there are no biomolecular reports concerning the relationship between Binswanger's disease and AD until now.

\section{Cerebral atrophy: a common morphological feature in both AD and CSVD}

Cerebral atrophy, a common feature of brain disorders, is a condition in which brain cells are lost, or the connections between them are damaged, resulting in the decrease of normal brain volume. ${ }^{105,106}$ A number of conditions can lead to brain atrophy, including epilepsy, ${ }^{107}$ traumatic brain injuries, ${ }^{108,109}$ strokes, ${ }^{110} \mathrm{AD},{ }^{105}$ multiple sclerosis, ${ }^{111}$ cerebral palsy, ${ }^{112}$ and Huntington's disease. ${ }^{113}$ Like other atrophies, cerebral atrophy involves loss of tissue. Loss of brain tissue can lead to sinister consequences, including a variety of neurological and cognitive problems. Focal cerebral atrophy, the corresponding damage concerned with a particular area of the brain, manifests the corresponding function impairment of concerned area of brain. Generalized cerebral atrophy will be linked with a range of clinical problems because of the involvement of the whole brain.

Cerebral atrophy can usually be identified in medical imaging such as Computed Tomography (CT) and/or MRI, which can demonstrate structural changes in the brain. Increasing imaging results have evidenced that CSVD has a close association with cerebral atrophy. ${ }^{114,115}$ A prospective follow-up research conducted by Nitkunan et al showed that brain volume is decreased in the patients with CSVD with respect to normal aging subjects, and this decrease was correlated with cognition decline during 1-year prospective follow-up. ${ }^{116}$ The leukoaraiosis and disability study further proved that brain atrophy facilitates cognitive decline, and brain atrophy is independently related to longitudinal cognitive decline in CSVD. ${ }^{117}$ According to MRI measurement, the severity of brain atrophy was correlated with the number of lacunar infarcts and the size of subcortical and periventricular white matter lesions, while shorter telomere length is associated with brain atrophy and WMH. ${ }^{114,118-120}$

A large number of imaging studies have confirmed that cerebral atrophy is the most significant morphological characteristic of $\mathrm{AD},{ }^{121-125}$ which has the widening grooves and fissures of the cerebral cortex, indicating progressively severe brain atrophy and loss of brain mass. It has been found that the acceleration of hippocampal atrophy in mild cognitive impairment subjects enhances the progress to clinical AD within 3 years of baseline, ${ }^{105}$ and regional measures of hippocampal atrophy are the strongest predictors of progression to AD. ${ }^{126}$

On the basis of the above information, it can be suggested that cerebral atrophy is a common morphologic feature in both AD and CSVD. On the background of cerebral atrophy, what is the relationship between AD and CSVD? The problem is still pending. Thus, it is important to reveal the exact role of CSVD in AD underlying cerebral atrophy.

\section{Dementia: a common clinical result of both AD and CSVD}

CSVD is common in the elderly and enhances the process of cognitive impairment and dementia. ${ }^{127,128}$ Clinical studies implicate the link between cognitive disorders with white matter lesions (a marker of small vessel disease in the brain) caused by aging, hypertension, systemic circulatory disturbances, or other processes (cerebral amyloid angiopathy, cerebral autosomal dominant arteriopathy with subcortical infarcts, and leukoencephalopathy). ${ }^{129-131}$ Genetic predispositions and environmental exposures may promote the development of CSVD and interact with normal aging to impact cognitive function. ${ }^{132,133}$ It has been demonstrated that 
the increasing severity of white matter lesions was associated with a steeper decline in cognitive function, accompanying with generalized brain atrophy and the presence of brain infarcts. ${ }^{127,134}$ White matter diffuse lesion could directly influence the recall processes controlled by the frontal lobe. ${ }^{135}$ The cognitive impairment is specifically focused on information processing speed and executive function in older people, implicating that CSVD may lead to cognitive impairment by blemishing information processing speed and executive function. ${ }^{127}$ The epidemiology of dementia in Singapore study showed that cerebral microbleeds were, independent of other concomitant markers of CSVD, helpful in poorer cognitive function. ${ }^{136}$

Available evidence demonstrates that cognitive impairment and dementia are the clinical manifestation for both AD and CSVD. ${ }^{137,138}$ Increasing evidence of a complex relationship between $\mathrm{AD}$ and cognitive impairment and $\mathrm{VaD}$ has been reported while CVD and stroke are related to high risk of both cognitive impairment and AD. ${ }^{139,140}$ The manifestation of CVD in imaging is extremely similar to AD in the elderly, whereas imaging markers of subcortical vascular diseases (leukoaraiosis, lacunar infarcts, microbleeds, ventricular enlargement, cortical, and hippocampal atrophy). ${ }^{141-143}$ Leukoaraiosis is linked with functional impairment in older patients with $\mathrm{AD}$ but not $\mathrm{VaD}$, while leukoaraiosis might play a synergistic effect with cognitive and behavioral disturbances to the onset and progression of cognitive disability of AD. ${ }^{144}$ The Sunnybrook Dementia Study demonstrated that Visible Virchow-Robin spaces in the white matter, markers for small vessel diseases, may be more related to $\mathrm{AD}$-related vascular pathology since $\mathrm{AD}$ had significantly greater volumes of $\mathrm{WMH}$, lacunes, and Virchow-Robin spaces in the white matter, ${ }^{145}$ and $\mathrm{WMH}$ may be related to regional neurodegeneration. ${ }^{146,147}$ It has been described and illustrated that WMH volume, particularly in parietal regions, is elevated among individuals with risk for $\mathrm{AD}$, predicts future diagnosis of $\mathrm{AD}$, predicts the rate of progression of cognitive symptoms among individuals with AD, and increases over time among individuals destined to develop AD. ${ }^{148}$ The vascular pathology of CSVD in $\mathrm{AD}$ may interact with neurodegenerative process and deteriorate cognitive decline. Thus, the progress of disorders from cognitive impairment to dementia can be attributed to cerebrovascular causes. ${ }^{149-151}$

\section{Conclusion and perspective}

CSVD has been readily identified on CT and/or MRI scans. CSVD, a leading cause of cognitive impairment, ultimately leads to dementia and contributes to neurodegenerative progress, including Alzheimer's onset. Up to now, a number of studies have confirmed that CSVD can co-occur with $\mathrm{AD}$, and signs of AD pathology have been found in CSVD, indicating that the pathology of CSVD and AD are interconnected. It seems that CSVD could stimulate amyloid pathology, while AD-associated cerebral amyloid pathology may enhance auxiliary vascular damage. The association between CSVD and incident dementia and Alzheimer's onset has never been well clarified. CSVD has been highlighted as a potential key risk factor for $\mathrm{AD}$, linking the main pathological contributors of $A \beta$ accumulation with cerebrovascular damage. However, there are no biomolecular reports concerning the relationship Binswanger's disease and AD until now. The role of cerebral microbleeds in $\mathrm{AD}$ remains to be identified. Furthermore, it remains unclear if and how associations between CSVD and Alzheimer's pathology result in cognitive impairment and dementia.

According to the above information, it can be suggested that CSVD may play a crucial role in AD. Based on the theory of CVD, the treatment and prevention for CVDs will benefit AD. It seems that statins have such a role in the treatment and prevention of Alzheimer's neurodegeneration since they can benefit CVDs. In spite of this, there is no strong evidencebased medicine to support the idea. So far, increasing basic molecular biology findings report that the treatment and prevention for CVDs will benefit AD. However, there is still lack of evidence in clinical application involved in specific drugs to benefit both AD and CSVD.

\section{Acknowledgments}

This work was supported by the Natural Science Foundation of Hubei Province (2015CFB260), the Hubei Province Health and Family Planning Scientific Research Project (WJ2015MB219), the Shiyan Natural Science Foundation (15K70), and the Natural Science Foundation of Renmin Hospital, Hubei University of Medicine to Dr Zhiyou Cai.

\section{Disclosure}

The authors report no conflicts of interest in this work.

\section{References}

1. Vinters HV. Emerging concepts in Alzheimer's disease. Annu Rev Pathol. 2015;10:291-319.

2. Wurtman R. Biomarkers in the diagnosis and management of Alzheimer's disease. Metabolism. 2015;64(3 suppl 1):S47-S50.

3. Bloom GS. Amyloid-beta and tau: the trigger and bullet in Alzheimer disease pathogenesis. JAMA Neurol. 2014;71(4):505-508.

4. Cai Z. Monoamine oxidase inhibitors: promising therapeutic agents for Alzheimer's disease (Review). Mol Med Rep. 2014;9(5):1533-1541. 
5. Tomimoto H. [Vascular cognitive impairment: the relationship between hypertensive small vessel disease and cerebral amyloid angiopathy]. Brain Nerve. 2012;64(12):1377-1386. Japanese.

6. de la Torre JC. Cardiovascular risk factors promote brain hypoperfusion leading to cognitive decline and dementia. Cardiovasc Psychiatry Neurol. 2012;2012:367516.

7. Pasquier F, Boulogne A, Leys D, Fontaine P. Diabetes mellitus and dementia. Diabetes Metab. 2006;32(5 pt 1):403-414.

8. Palacio S, McClure LA, Benavente OR, Bazan C 3rd, Pergola P, Hart RG. Lacunar strokes in patients with diabetes mellitus: risk factors, infarct location, and prognosis: the secondary prevention of small subcortical strokes study. Stroke. 2014;45(9):2689-2694.

9. Zheng L, Vinters HV, Mack WJ, Zarow C, Ellis WG, Chui HC. Cerebral atherosclerosis is associated with cystic infarcts and microinfarcts but not Alzheimer pathologic changes. Stroke. 2013;44(10): 2835-2841.

10. Siegel GJ, Chauhan NB, Feinstein DL, et al. Statin therapy is associated with reduced neuropathologic changes of Alzheimer disease. Neurology. 2008;71(5):383. Author reply 383.

11. Zhang M, Chen M, Wang Q, et al. Relationship between cerebral microbleeds and cognitive function in lacunar infarct. $J$ Int Med Res. 2013; 41(2):347-355.

12. Lammie GA, Brannan F, Slattery J, Warlow C. Nonhypertensive cerebral small-vessel disease. An autopsy study. Stroke. 1997;28(11): 2222-2229.

13. Schmidt H, Zeginigg M, Wiltgen M, et al. Genetic variants of the NOTCH3 gene in the elderly and magnetic resonance imaging correlates of age-related cerebral small vessel disease. Brain. 2011;134(pt 11) 3384-3397.

14. Paquet C, Jouvent E, Mine M, et al. A cortical form of CADASIL with cerebral Abeta amyloidosis. Acta Neuropathol. 2010;120(6): 813-820.

15. Pantoni L. Cerebral small vessel disease: from pathogenesis and clinical characteristics to therapeutic challenges. Lancet Neurol. 2010; 9(7):689-701

16. Schmidtke K, Hull M. Cerebral small vessel disease: how does it progress? J Neurol Sci. 2005;22(9-230):13-20.

17. Zhang AJ, Yu XJ, Wang M. The clinical manifestations and pathophysiology of cerebral small vessel disease. Neurosci Bull. 2010;26(3): 257-264.

18. Lammie GA. Hypertensive cerebral small vessel disease and stroke. Brain Pathol. 2002;12(3):358-370.

19. Onodera O. [What is cerebral small vessel disease?]. Rinsho Shinkeigaku. 2011;51(6):399-405. Japanese.

20. Yates PA, Villemagne VL, Ellis KA, Desmond PM, Masters CL, Rowe CC. Cerebral microbleeds: a review of clinical, genetic, and neuroimaging associations. Front Neurol. 2014;4:205.

21. Iadecola C. Rescuing troubled vessels in Alzheimer disease. Nat Med. 2005;11(9):923-924.

22. Iadecola $\mathrm{C}$. The overlap between neurodegenerative and vascular factors in the pathogenesis of dementia. Acta Neuropathol. 2010; 120(3):287-296.

23. Lockhart A, Lamb JR, Osredkar T, et al. PIB is a non-specific imaging marker of amyloid-beta (Abeta) peptide-related cerebral amyloidosis Brain. 2007;130(pt 10):2607-2615.

24. Iadecola C. Cerebrovascular effects of amyloid-beta peptides: mechanisms and implications for Alzheimer's dementia. Cell Mol Neurobiol. 2003;23(4-5):681-689.

25. Roher AE, Kuo YM, Esh C, et al. Cortical and leptomeningeal cerebrovascular amyloid and white matter pathology in Alzheimer's disease. Mol Med. 2003;9(3-4):112-122.

26. Brown WR, Thore CR. Review: cerebral microvascular pathology in ageing and neurodegeneration. Neuropathol Appl Neurobiol. 2011 37(1):56-74.

27. Roher AE, Esh C, Kokjohn TA, et al. Circle of Willis atherosclerosis is a risk factor for sporadic Alzheimer's disease. Arterioscler Thromb Vasc Biol. 2003;23(11):2055-2062.
28. Yarchoan M, Xie SX, Kling MA, et al. Cerebrovascular atherosclerosis correlates with Alzheimer pathology in neurodegenerative dementias. Brain. 2012;135(pt 12):3749-3756.

29. Girouard H, Iadecola C. Neurovascular coupling in the normal brain and in hypertension, stroke, and Alzheimer disease. J Appl Physiol (1985). 2006;100(1):328-335.

30. Henry-Feugeas MC. Alzheimer's disease in late-life dementia: a minor toxic consequence of devastating cerebrovascular dysfunction. Med Hypotheses. 2008;70(4):866-875.

31. Sacco S, Marini C, Totaro R, Russo T, Cerone D, Carolei A. A population-based study of the incidence and prognosis of lacunar stroke. Neurology. 2006;66(9):1335-1338.

32. Lastilla M. Lacunar infarct. Clin Exp Hypertens. 2006;28(3-4): 205-215.

33. Das T, Settecase F, Boulos M, et al. Multimodal CT provides improved performance for lacunar infarct detection. AJNR Am J Neuroradiol. 2015;36(6):1069-1075.

34. Labovitz DL, Boden-Albala B, Hauser WA, Sacco RL. Lacunar infarct or deep intracerebral hemorrhage: who gets which? The Northern Manhattan study. Neurology. 2007;68(8):606-608.

35. Masana Y, Iwamoto F, Yamada M, Motozaki T, Yoshimine T. [Prevalence and risk factors for silent lacunar infarct in white matter lesion - brain multiphasic screening]. No To Shinkei. 2003;55(12): 1027-1032. Japanese.

36. Arboix A. Lacunar infarct and cognitive decline. Expert Rev Neurother 2011;11(9):1251-1254.

37. van Zandvoort MJ, van der Grond J, Kappelle LJ, de Haan EH. Cognitive deficits and changes in neurometabolites after a lacunar infarct. J Neurol. 2005;252(2):183-190.

38. Chen CF, Lan SH, Khor GT, Lai CL, Tai CT. Cognitive dysfunction after acute lacunar infarct. Kaohsiung J Med Sci. 2005;21(6):267-271.

39. Grinberg LT, Thal DR. Vascular pathology in the aged human brain. Acta Neuropathol. 2010;119(3):277-290.

40. Benisty S, Gouw AA, Porcher R, et al. Location of lacunar infarcts correlates with cognition in a sample of non-disabled subjects with agerelated white-matter changes: the LADIS study. J Neurol Neurosurg Psychiatry. 2009;80(5):478-483.

41. Wen HM, Mok VC, Fan YH, et al. Effect of white matter changes on cognitive impairment in patients with lacunar infarcts. Stroke. 2004; 35(8):1826-1830

42. Nagata K, Takano D, Yamazaki T, et al. Cerebrovascular lesions in elderly Japanese patients with Alzheimer's disease. J Neurol Sci. 2012; 322(1-2):87-91.

43. Whitehead SN, Massoni E, Cheng G, et al. Triflusal reduces cerebral ischemia induced inflammation in a combined mouse model of Alzheimer's disease and stroke. Brain Res. 2010;1366:246-256.

44. Richard E, Gouw AA, Scheltens P, van Gool WA. Vascular care in patients with Alzheimer disease with cerebrovascular lesions slows progression of white matter lesions on MRI: the evaluation of vascular care in Alzheimer's disease (EVA) study. Stroke. 2010;41(3): 554-556.

45. Gold G, Giannakopoulos P, Herrmann FR, Bouras C, Kovari E. Identification of Alzheimer and vascular lesion thresholds for mixed dementia. Brain. 2007;130(pt 11):2830-2836

46. Snowdon DA, Greiner LH, Mortimer JA, Riley KP, Greiner PA, Markesbery WR. Brain infarction and the clinical expression of Alzheimer disease. The Nun study. JAMA. 1997;277(10):813-817.

47. Heyman A, Fillenbaum GG, Welsh-Bohmer KA, et al. Cerebral infarcts in patients with autopsy-proven Alzheimer's disease: CERAD, part XVIII. Consortium to establish a registry for Alzheimer's disease. Neurology. 1998;51(1):159-162.

48. van Rooden S, Goos JD, van Opstal AM, et al. Increased number of microinfarcts in Alzheimer disease at 7-T MR imaging. Radiology. 2014;270(1):205-211.

49. Kester MI, Goos JD, Teunissen CE, et al. Associations between cerebral small-vessel disease and Alzheimer disease pathology as measured by cerebrospinal fluid biomarkers. JAMA Neurol. 2014;71(7):855-862. 
50. Erten-Lyons D, Woltjer R, Kaye J, et al. Neuropathologic basis of white matter hyperintensity accumulation with advanced age. Neurology. 2013;81(11):977-983.

51. Gurol ME, Irizarry MC, Smith EE, et al. Plasma beta-amyloid and white matter lesions in AD, MCI, and cerebral amyloid angiopathy. Neurology. 2006;66(1):23-29.

52. De Reuck JL, Cordonnier C, Deramecourt V, et al. Microbleeds in postmortem brains of patients with Alzheimer disease: a T2*-weighted gradient-echo 7.0 T magnetic resonance imaging study. Alzheimer Dis Assoc Disord. 2013;27(2):162-167.

53. Yakushiji Y, Hara H. [Cerebral microbleeds: clinical features and management]. Rinsho Shinkeigaku. 2012;52(11):1106-1109. Japanese.

54. Park JH, Seo SW, Kim C, et al. Pathogenesis of cerebral microbleeds: in vivo imaging of amyloid and subcortical ischemic small vessel disease in 226 individuals with cognitive impairment. Ann Neurol. 2013;73(5):584-593.

55. Ghelmez D, Sorin Tuta S, Popa C. Cerebral microbleeds (CMBs) relevance for mechanisms of cerebral hemorrhage - analysis of $24 \mathrm{MRI}$ evaluated patients. J Med Life. 2013;6(4):437-439.

56. Shams S, Martola J, Granberg T, et al. Cerebral microbleeds: different prevalence, topography, and risk factors depending on dementia diagnosis - the Karolinska Imaging Dementia Study. AJNR Am J Neuroradiol. 2015;36(4):661-666.

57. Nagasawa J, Kiyozaka T, Ikeda K. Prevalence and clinicoradiological analyses of patients with Alzheimer disease coexisting multiple microbleeds. J Stroke Cerebrovasc Dis. 2014;23(9):2444-2449.

58. Goos JD, Kester MI, Barkhof F, et al. Patients with Alzheimer disease with multiple microbleeds: relation with cerebrospinal fluid biomarkers and cognition. Stroke. 2009;40(11):3455-3460.

59. Heringa SM, Reijmer YD, Leemans A, Koek HL, Kappelle LJ, Biessels GJ. Multiple microbleeds are related to cerebral network disruptions in patients with early Alzheimer's disease. $J$ Alzheimers Dis. 2014;38(1):211-221.

60. Whitwell JL, Kantarci K, Weigand SD, et al. Microbleeds in atypical presentations of Alzheimer's disease: a comparison to dementia of the Alzheimer's type. J Alzheimers Dis. 2015;45(4):1109-1117.

61. Nakata-Kudo Y, Mizuno T, Yamada K, et al. Microbleeds in Alzheimer disease are more related to cerebral amyloid angiopathy than cerebrovascular disease. Dement Geriatr Cogn Disord. 2006;22(1):8-14.

62. Martinez-Ramirez S, Greenberg SM, Viswanathan A. Cerebral microbleeds: overview and implications in cognitive impairment. Alzheimers Res Ther. 2014;6(3):33.

63. Stone J, Johnstone DM, Mitrofanis J, O'Rourke M. The mechanical cause of age-related dementia (Alzheimer's disease): the brain is destroyed by the pulse. J Alzheimers Dis. 2015;44(2):355-373.

64. Benedictus MR, Goos JD, Binnewijzend MA, et al. Specific risk factors for microbleeds and white matter hyperintensities in Alzheimer's disease. Neurobiol Aging. 2013;34(11):2488-2494.

65. Goos JD, Teunissen CE, Veerhuis R, et al. Microbleeds relate to altered amyloid-beta metabolism in Alzheimer's disease. Neurobiol Aging. 2012;33(5):1011.e1011-e1019.

66. Yates PA, Desmond PM, Phal PM, et al. Incidence of cerebral microbleeds in preclinical Alzheimer disease. Neurology. 2014;82(14): 1266-1273.

67. Olazaran J, Ramos A, Boyano I, et al. Pattern of and risk factors for brain microbleeds in neurodegenerative dementia. Am J Alzheimers Dis Other Demen. 2014;29(3):263-269.

68. Brundel M, Heringa SM, de Bresser J, et al. High prevalence of cerebral microbleeds at 7Tesla MRI in patients with early Alzheimer's disease. J Alzheimers Dis. 2012;31(2):259-263.

69. Wen Y, Miyashita A, Kitamura N, et al. SORL1 is genetically associated with neuropathologically characterized late-onset Alzheimer's disease. J Alzheimers Dis. 2013;35(2):387-394.

70. Feng X, Hou D, Deng Y, Li W, Tian M, Yu Z. SORL1 gene polymorphism association with late-onset Alzheimer's disease. Neurosci Lett. 2015;584:382-389.

71. Yin RH, Yu JT, Tan L. The role of SORL1 in Alzheimer's disease. Mol Neurobiol. 2015;51(3):909-918.
72. Xue X, Zhang M, Lin Y, Xu E, Jia J. Association between the SORL1 rs2070045 polymorphism and late-onset Alzheimer's disease: interaction with the ApoE genotype in the Chinese Han population. Neurosci Lett. 2014;559:94-98.

73. Felsky D, Szeszko P, Yu L, et al. The SORL1 gene and convergent neural risk for Alzheimer's disease across the human lifespan. Mol Psychiatry. 2014;19(10):1125-1132.

74. Louwersheimer E, Ramirez A, Cruchaga C, et al. The influence of genetic variants in SORL1 gene on the manifestation of Alzheimer's disease. Neurobiol Aging. 2015;36(3):1605.e1613-e1620.

75. Schuur M, van Swieten JC, Schol-Gelok S, et al. Genetic risk factors for cerebral small-vessel disease in hypertensive patients from a genetically isolated population. J Neurol Neurosurg Psychiatry. 2011; 82(1):41-44.

76. Cordonnier C. Brain microbleeds. Pract Neurol. 2010;10(2):94-100.

77. Loitfelder M, Seiler S, Schwingenschuh P, Schmidt R. Cerebral microbleeds: a review. Panminerva Med. 2012;54(3):149-160.

78. Hommet C, Mondon $\mathrm{K}$, Constans $\mathrm{T}$, et al. Review of cerebral microangiopathy and Alzheimer's disease: relation between white matter hyperintensities and microbleeds. Dement Geriatr Cogn Disord. 2011;32(6):367-378.

79. Cordonnier C, van der Flier WM. Brain microbleeds and Alzheimer's disease: innocent observation or key player? Brain. 2011;134(pt 2): 335-344.

80. van der Flier WM. Clinical aspects of microbleeds in Alzheimer's disease. J Neurol Sci. 2012;322(1-2):56-58.

81. O'Sullivan M. Leukoaraiosis. Pract Neurol. 2008;8(1):26-38.

82. Auriel E, Bornstein NM, Berenyi E, et al. Clinical, radiological and pathological correlates of leukoaraiosis. Acta Neurol Scand. 2011; 123(1):41-47.

83. Huang YH, Zhang WW, Lin L, et al. Could changes in arterioles impede the perivascular drainage of interstitial fluid from the cerebral white matter in leukoaraiosis? Neuropathol Appl Neurobiol. 2010;36(3): 237-247.

84. Uh J, Yezhuvath U, Cheng Y, Lu H. In vivo vascular hallmarks of diffuse leukoaraiosis. J Magn Reson Imaging. 2010;32(1):184-190.

85. Bogousslavsky J. [Leukoencephalopathy, leukoaraiosis and cerebral infarction]. Rev Neurol (Paris). 1988;144(1):11-17. Japanese.

86. Duron E, Vidal JS, Bounatiro S, et al. Relationships between personality traits, medial temporal lobe atrophy, and white matter lesion in subjects suffering from mild cognitive impairment. Front Aging Neurosci. 2014;6:195.

87. Pluta R, Januszewski S, Ulamek M. Ischemic blood-brain barrier and amyloid in white matter as etiological factors in leukoaraiosis. Acta Neurochir Suppl. 2008;102:353-356.

88. Moody DM, Brown WR, Challa VR, Ghazi-Birry HS, Reboussin DM. Cerebral microvascular alterations in aging, leukoaraiosis, and Alzheimer's disease. Ann N Y Acad Sci. 1997;826:103-116.

89. Coffman JA, Torello MW, Bornstein RA, Chakeres D, Burns E, Nasrallah HA. Leukoaraiosis in asymptomatic adult offspring of individuals with Alzheimer's disease. Biol Psychiatry. 1990;27(11):1244-1248.

90. Diaz JF, Merskey H, Hachinski VC, et al. Improved recognition of leukoaraiosis and cognitive impairment in Alzheimer's disease. Arch Neurol. 1991;48(10):1022-1025.

91. Sarabia-Cobo CM, Perez V, Hermosilla C, Nunez MJ, de Lorena P. Apathy and leukoaraiosis in mild cognitive impairment and Alzheimer's disease: multicenter diagnostic criteria according to the latest studies. Dement Geriatr Cogn Dis Extra. 2014;4(2):228-235.

92. Chung CP, Beggs C, Wang PN, et al. Jugular venous reflux and white matter abnormalities in Alzheimer's disease: a pilot study. J Alzheimers Dis. 2014;39(3):601-609.

93. Chen W, Song X, Zhang Y. Assessment of the Virchow-Robin spaces in Alzheimer disease, mild cognitive impairment, and normal aging, using high-field MR imaging. AJNR Am J Neuroradiol. 2011; 32(8):1490-1495.

94. Makedonov I, Black SE, MacIntosh BJ. Cerebral small vessel disease in aging and Alzheimer's disease: a comparative study using MRI and SPECT. Eur J Neurol. 2013;20(2):243-250. 
95. Verdelho A, Madureira S, Moleiro C, et al. Self-perceived memory complaints predict progression to Alzheimer disease. The LADIS study. J Alzheimers Dis. 2011;27(3):491-498.

96. van Swieten JC, Caplan LR. Binswanger's disease. Adv Neurol. 1993;62:193-211.

97. Schorer CE. Alzheimer and Kraepelin describe Binswanger's disease. J Neuropsychiatry Clin Neurosci. 1992;4(1):55-58.

98. Ferrer I, Bella R, Serrano MT, Marti E, Guionnet N. Arteriolosclerotic leucoencephalopathy in the elderly and its relation to white matter lesions in Binswanger's disease, multi-infarct encephalopathy and Alzheimer's disease. J Neurol Sci. 1990;98(1):37-50.

99. Peterson B, Summergrad P. Binswanger's disease (Part II): pathogenesis of subcortical arteriosclerotic encephalopathy and its relation to other dementing processes. J Geriatr Psychiatry Neurol. 1989;2(4):171-181.

100. Fisher CM. Binswanger's encephalopathy: a review. J Neurol. 1989; 236(2):65-79.

101. Sandyk R. Subcortical arteriosclerotic encephalopathy (Binswanger's disease). S Afr Med J. 1983;63(6):204-205.

102. Tomimoto H, Akiguchi I, Akiyama H, et al. Vascular changes in white matter lesions of Alzheimer's disease. Acta Neuropathol. 1999; 97(6):629-634.

103. Kosaka K, Ikeda K, Matsushita M, Iizuka R. A combination of Alzheimer's and Binswanger's diseases - a clinicopathological study of four cases. Jpn J Psychiatry Neurol. 1986;40(4):685-692.

104. Watanabe T, Shiino A, Akiguchi I. Absolute quantification in proton magnetic resonance spectroscopy is superior to relative ratio to discriminate Alzheimer's disease from Binswanger's disease. Dement Geriatr Cogn Disord. 2008;26(1):89-100

105. Leung KK, Bartlett JW, Barnes J, Manning EN, Ourselin S, Fox NC Cerebral atrophy in mild cognitive impairment and Alzheimer disease: rates and acceleration. Neurology. 2013;80(7):648-654.

106. Beck C, Kruetzelmann A, Forkert ND, et al. A simple brain atrophy measure improves the prediction of malignant middle cerebral artery infarction by acute DWI lesion volume. J Neurol. 2014;261(6): 1097-1103.

107. Henny C, Despland PA, Regli F. [Initial epileptic crisis after the age of 60: etiology, clinical aspects and EEG]. Schweiz Med Wochenschr. 1990;120(21):787-792. Japanese.

108. Bigler ED. The clinical significance of cerebral atrophy in traumatic brain injury. Arch Clin Neuropsychol. 1987;2(3):293-304.

109. Tate DF, Khedraki R, Neeley ES, Ryser DK, Bigler ED. Cerebral volume loss, cognitive deficit, and neuropsychological performance: comparative measures of brain atrophy: II. Traumatic brain injury J Int Neuropsychol Soc. 2011;17(2):308-316.

110. Aoi MC, Hu K, Lo MT, Selim M, Olufsen MS, Novak V. Impaired cerebral autoregulation is associated with brain atrophy and worse functional status in chronic ischemic stroke. PLoS One. 2012;7(10): e46794

111. Dell'Oglio E, Ceccarelli A, Glanz BI, et al. Quantification of global cerebral atrophy in multiple sclerosis from 3T MRI using SPM: the role of misclassification errors. J Neuroimaging. 2015;25(2): 191-199.

112. Cordato NJ, Duggins AJ, Halliday GM, Morris JG, Pantelis C. Clinical deficits correlate with regional cerebral atrophy in progressive supranuclear palsy. Brain. 2005;128(pt 6):1259-1266.

113. Kassubek J, Bernhard Landwehrmeyer G, Ecker D, et al. Global cerebral atrophy in early stages of Huntington's disease: quantitative MRI study. Neuroreport. 2004;15(2):363-365.

114. Aribisala BS, Valdes Hernandez MC, Royle NA, et al. Brain atrophy associations with white matter lesions in the ageing brain: the Lothian Birth Cohort 1936. Eur Radiol. 2013;23(4):1084-1092.

115. Sala S, Agosta F, Pagani E, Copetti M, Comi G, Filippi M. Microstructural changes and atrophy in brain white matter tracts with aging. Neurobiol Aging. 2012;33(3):488-498.e482.

116. Nitkunan A, Lanfranconi S, Charlton RA, Barrick TR, Markus HS. Brain atrophy and cerebral small vessel disease: a prospective follow-up study. Stroke. 2011;42(1):133-138.
117. Jokinen H, Lipsanen J, Schmidt R, et al. Brain atrophy accelerates cognitive decline in cerebral small vessel disease: the LADIS study. Neurology. 2012;78(22):1785-1792.

118. Thong JY, Hilal S, Wang Y, et al. Association of silent lacunar infarct with brain atrophy and cognitive impairment. J Neurol Neurosurg Psychiatry. 2013;84(11):1219-1225.

119. Wikgren M, Karlsson T, Soderlund H, et al. Shorter telomere length is linked to brain atrophy and white matter hyperintensities. Age Ageing. 2014;43(2):212-217

120. Kanhai DA, de Kleijn DP, Kappelle LJ, et al. Extracellular vesicle protein levels are related to brain atrophy and cerebral white matter lesions in patients with manifest vascular disease: the SMART-MR study. BMJ Open. 2014;4(1):e003824.

121. Guo H, Song X, Vandorpe R, et al. Evaluation of common structural brain changes in aging and Alzheimer disease with the use of an MRI-based brain atrophy and lesion index: a comparison between T1WI and T2WI at 1.5T and 3T. AJNR Am J Neuroradiol. 2014;35(3):504-512.

122. Anandh KR, Sujatha CM, Ramakrishnan S. Atrophy analysis of corpus callosum in Alzheimer brain MR images using anisotropic diffusion filtering and level sets. Conf Proc IEEE Eng Med Biol Soc. 2014;2014:1945-1948.

123. Whitwell JL, Jack CR Jr, Pankratz VS, et al. Rates of brain atrophy over time in autopsy-proven frontotemporal dementia and Alzheimer disease. Neuroimage. 2008;39(3):1034-1040.

124. Sluimer JD, Vrenken H, Blankenstein MA, et al. Whole-brain atrophy rate in Alzheimer disease: identifying fast progressors. Neurology. 2008;70(19 pt 2):1836-1841.

125. Bokde AL, Pietrini P, Ibanez V, et al. The effect of brain atrophy on cerebral hypometabolism in the visual variant of Alzheimer disease. Arch Neurol. 2001;58(3):480-486.

126. Henneman WJ, Sluimer JD, Barnes J, et al. Hippocampal atrophy rates in Alzheimer disease: added value over whole brain volume measures. Neurology. 2009;72(11):999-1007.

127. Prins ND, van Dijk EJ, den Heijer T, et al. Cerebral small-vessel disease and decline in information processing speed, executive function and memory. Brain. 2005;128(pt 9):2034-2041.

128. Sierra C. Cerebral small vessel disease, cognitive impairment and vascular dementia. Panminerva Med. 2012;54(3):179-188.

129. Pantoni L, Garcia JH. Cognitive impairment and cellular/vascular changes in the cerebral white matter. Ann N Y Acad Sci. 1997;826:92-102.

130. Richardson K, Stephan BC, Ince PG, Brayne C, Matthews FE, Esiri MM. The neuropathology of vascular disease in the medical research council cognitive function and ageing study (MRC CFAS) Curr Alzheimer Res. 2012;9(6):687-696.

131. Costanza A, Xekardaki A, Kovari E, Gold G, Bouras C, Giannakopoulos P. Microvascular burden and Alzheimer-type lesions across the age spectrum. J Alzheimers Dis. 2012;32(3):643-652.

132. Rincon F, Wright CB. Current pathophysiological concepts in cerebral small vessel disease. Front Aging Neurosci. 2014;6:24.

133. Meier IB, Manly JJ, Provenzano FA, et al. White matter predictors of cognitive functioning in older adults. J Int Neuropsychol Soc. 2012; 18(3):414-427.

134. Pasquier F, Leys D. Why are stroke patients prone to develop dementia? J Neurol. 1997;244(3):135-142.

135. Suades-Gonzalez E, Jodar-Vicente M, Perdrix-Solas D. [Memory deficit in patients with subcortical vascular cognitive impairment versus Alzheimer-type dementia: the sensitivity of the 'word list' subtest on the Wechsler Memory Scale-III]. Rev Neurol. 2009; 49(12):623-629. Japanese.

136. Hilal S, Saini M, Tan CS, et al. Cerebral microbleeds and cognition: the epidemiology of dementia in Singapore study. Alzheimer Dis Assoc Disord. 2014;28(2):106-112.

137. De Deyn PP, Goeman J, Engelborghs S, et al. From neuronal and vascular impairment to dementia. Pharmacopsychiatry. 1999;32(suppl 1): $17-24$.

138. Kovacic JC, Fuster V. Atherosclerotic risk factors, vascular cognitive impairment, and Alzheimer disease. Mt Sinai J Med. 2012;79(6):664-673. 
139. Erkinjuntti T. Diagnosis and management of vascular cognitive impairment and dementia. J Neural Transm Suppl. 2002;(63):91-109.

140. Attems J, Jellinger KA. The overlap between vascular disease and Alzheimer's disease - lessons from pathology. BMC Med. 2014; 12:206.

141. Henry-Feugeas MC. MRI of the 'Alzheimer syndrome'. J Neuroradiol. 2007;34(4):220-227.

142. Cechetto DF, Hachinski V, Whitehead SN. Vascular risk factors and Alzheimer's disease. Expert Rev Neurother. 2008;8(5):743-750.

143. Chalmers K, Wilcock G, Love S. Contributors to white matter damage in the frontal lobe in Alzheimer's disease. Neuropathol Appl Neurobiol. 2005;31(6):623-631.

144. Ble A, Ranzini M, Zurlo A, et al. Leukoaraiosis is associated with functional impairment in older patients with Alzheimer's disease but not vascular dementia. $J$ Nutr Health Aging. 2006;10(1):31-35.

145. Ramirez J, Berezuk C, McNeely AA, Scott CJ, Gao F, Black SE. Visible Virchow-Robin spaces on magnetic resonance imaging of Alzheimer's disease patients and normal elderly from the Sunnybrook Dementia Study. J Alzheimers Dis. 2015;43(2):415-424.
146. Guzman VA, Carmichael OT, Schwarz C, Tosto G, Zimmerman ME, Brickman AM. White matter hyperintensities and amyloid are independently associated with entorhinal cortex volume among individuals with mild cognitive impairment. Alzheimers Dement. 2013; 9(5 suppl):S124-S131.

147. Umegaki H. Pathophysiology of cognitive dysfunction in older people with type 2 diabetes: vascular changes or neurodegeneration? Age Ageing. 2010;39(1):8-10.

148. Brickman AM. Contemplating Alzheimer's disease and the contribution of white matter hyperintensities. Curr Neurol Neurosci Rep. 2013;13(12):415.

149. Klohs J, Rudin M, Shimshek DR, Beckmann N. Imaging of cerebrovascular pathology in animal models of Alzheimer's disease. Front Aging Neurosci. 2014;6:32.

150. Jellinger KA. Pathogenesis and treatment of vascular cognitive impairment. Neurodegener Dis Manag. 2014;4(6):471-490.

151. Targosz-Gajniak M, Siuda J, Ochudlo S, Opala G. Cerebral white matter lesions in patients with dementia - from MCI to severe Alzheimer's disease. J Neurol Sci. 2009;283(1-2):79-82.
Clinical Interventions in Aging

\section{Publish your work in this journal}

Clinical Interventions in Aging is an international, peer-reviewed journal focusing on evidence-based reports on the value or lack thereof of treatments intended to prevent or delay the onset of maladaptive correlates of aging in human beings. This journal is indexed on PubMed Central, MedLine,

\section{Dovepress}

CAS, Scopus and the Elsevier Bibliographic databases. The manuscript management system is completely online and includes a very quick and fair peer-review system, which is all easy to use. Visit http://www.dovepress. com/testimonials.php to read real quotes from published authors. 